\title{
Ultrasound-Enhanced Thrombolysis in Acute Ischemic Stroke: Potential, Failures, and Safety
}

\author{
Georgios Tsivgoulis and Andrei V. Alexandrov \\ Comprehensive Stroke Center, Department of Neurology, University of Alabama at Birmingham, Birmingham, Alabama 35294
}

\begin{abstract}
Summary: Experimental and pilot clinical evidence shows that thrombolysis with intravenous tissue plasminogen activator (TPA) can be enhanced with ultrasound. Ultrasound delivers mechanical pressure waves to the clot, thus exposing more thrombus surface to circulating drug. The international multicenter phase II CLOTBUST trial showed that, in patients with acute ischemic stroke, transcranial Doppler (TCD) monitoring augments TPA-induced arterial recanalization, with a nonsignificant trend toward an increased rate of recovery from stroke, compared with placebo. In the CLOTBUST trial, the dramatic clinical recovery from stroke coupled with complete recanalization within 2 hours after TPA bolus occurred in $25 \%$ of patients treated with TPA + TCD $(n=63)$, compared with $8 \%$ of those who received TPA alone $(n=63, P=0.02)$. Different results were achieved in smaller studies that used transcranial color-coded duplex sonography (TCCD) and a nonimaging
\end{abstract}

therapeutic ultrasound system. The findings of the TRUMBI trial (26 patients) underscored the adverse bioeffects of midkilohertz $(300 \mathrm{kHz})$ ultrasound, such as promotion of bleeding in brain areas both affected and unaffected by ischemia. Exposure to multifrequency, multielement duplex ultrasound resulted in a trend toward a higher risk of hemorrhagic transformation. To further enhance the ability of TPA to break up thrombi, current ongoing clinical trials include phase II studies of a single-beam, 2-MHz TCD with perflutren lipid microspheres. Enhancement of intra-arterial TPA delivery is being clinically tested with $1.7-2.1 \mathrm{MHz}$ pulsed-wave ultrasound (EKOS catheter). Multinational dose escalation studies of microspheres and the development of an operator-independent ultrasound device are underway. Key Words: Tissue plasminogen activator, transcranial Doppler, stroke, thrombolysis, microspheres.

\section{THROMBOLYSIS IN ACUTE CEREBRAL ISCHEMIA}

Unlike thrombolysis for myocardial ischemia, the pilot clinical studies of thrombolysis for ischemic stroke did not document any dramatic Lazarus or on-the-table clinical recovery during treatment. ${ }^{1-3}$ Subsequent pivotal trials of tissue plasminogen activator (TPA) have not reported any differences between the groups at 2 and 24 hours post treatment in the prespecified endpoints. ${ }^{4-7}$ However, a post hoc analysis of the NINDS trial showed that by 24 hours, $27 \%$ of TPA-treated patients improved by $\geq 10$ points on the National Institutes of Health Stroke Scale (NIHSS) or resolved their neurologic deficit completely, compared with $12 \%$ in the placebo group. ${ }^{8}$ Therefore, some patients may have experienced early clinical recovery, presumably due to fast thrombus dissolution, but the overall number of these events was low.

Address correspondence and reprint requests to: Andrei V. Alexandrov, MD, Comprehensive Stroke Center, Department of Neurology, University of Alabama at Birmingham, Suite 226, RWUHM, 1719 6th Avenue South, Birmingham, AL 35294. E-mail: avalexandrov@ att.net.
Early clinical improvement after stroke usually occurs after arterial recanalization. ${ }^{9-12}$ A recent meta-analysis confirmed the recanalization hypothesis by showing that recanalization is associated with a four- to fivefold increase in the odds of a good final functional outcome and a four- to fivefold reduction in the odds of death. ${ }^{13}$ These results lend strong support to the use of restoration of vessel patency as a surrogate endpoint in phase II trials of neurotherapeutic recanalization agents and in trials comparing novel to existing recanalization devices in acute ischemic stroke. Because early recanalization can lead to dramatic recovery, any additional enhancement of TPAassociated thrombus dissolution can possibly produce even higher early recovery rates among patients with ischemic stroke.

\section{EXPERIMENTAL EVIDENCE FOR ENHANCEMENT OF THROMBOLYSIS WITH ULTRASOUND}

The ability of ultrasonic mechanical pressure waves to enhance thrombolysis was first documented in 1976 and $1981,{ }^{14,15}$ and was confirmed by several investigators in 
experimental models. ${ }^{16-20}$ Various ultrasound energies $\left(0.2-2.0 \mathrm{~W} / \mathrm{cm}^{2}\right)$ and frequencies $(20 \mathrm{kHz}$ to $2 \mathrm{MHz})$ were used in these studies. Ultrasound can promote the motion of fluid around the thrombus, an effect called microstreaming. ${ }^{21}$ It is possible that the application of ultrasound energy agitates the blood close to the occluding thrombus and promotes the mixing of TPA, effectively increasing the concentration of the agent that is in contact with the thrombus. Consequently, it has been hypothesized that, in stroke patients, ultrasound can promote TPA delivery to the areas with stagnant flow near the occlusion.

The pressure waves generated by ultrasound may also increase the permeation of TPA into the interior of the fibrin network. In addition, ultrasound waves can have direct effects on the binding of TPA to the fibrin mesh that forms the occlusive lesion. Ultrasound energy enhances the binding of TPA in vitro to the cross-linked fibrin and fibrin elements within a matrix,${ }^{22}$ and at the same time weakens the fibrin cross-links.

Although low kilohertz frequencies better potentiate TPA effects, ${ }^{23}$ these systems are not available for clinical practice because of safety concerns and the inability to image vasculature with this frequency/wavelength range. Meanwhile, frequencies of $1-2.2 \mathrm{MHz}$ can also enhance TPA-induced thrombus dissolution through various mechanisms, such as fluid streaming around clot surface, disaggregation of fibrin fibers, and creating more binding sites for TPA without heating or cavitation. ${ }^{24,25}$ This frequency range is safely and routinely used for diagnostic ultrasound examinations.

\section{DIAGNOSTIC APPLICATIONS OF ULTRASOUND IN ACUTE CEREBRAL ISCHEMIA}

Portable diagnostic 2-MHz transcranial Doppler (TCD) equipment can be used in the emergency room to continuously monitor TPA infusion in acute ischemic stroke patients. ${ }^{26} \mathrm{With}$ prior training and experience in the interpretation of TCD, this test, particularly in combination with urgent carotid and vertebral duplex scanning, can yield a high degree of accuracy for bedside detection and localization of arterial occlusion and recanalization. ${ }^{26,27}$ In addition, TCD can be complementary to other imaging modalities, such as computed tomographic (CT) angiography by showing real-time flow findings (e.g., embolization, collateralization of flow with extracranial internal carotid artery disease, alternating flow signals indicative of steal phenomenon). ${ }^{28} \mathrm{Fi}$ nally, real-time flow findings during TCD-monitoring are predictive of the long-term functional outcome in ischemic stroke. ${ }^{29,30}$

Once abnormal residual flow signals are identified, an ultrasound beam can be steadily focused at the presumed intracranial thrombus location, and arterial recanalization can be monitored in real time. ${ }^{26}$ In work at our institution, intravenous TPA infusion was continuously monitored with $2-\mathrm{MHz} \mathrm{TCD}^{26}$ and early recanalization and dramatic recovery rates higher than expected were observed. $^{26}$ Two other studies conducted by separate groups in Germany ${ }^{31}$ and France $^{32}$ also reported higher rates of recanalization than expected in patients with acute middle cerebral artery occlusion who were not eligible for TPA and received 2-MHz transcranial colorcoded Doppler continuous monitoring. These provocative findings suggest a potential therapeutic effect of diagnostic transcranial ultrasound in the setting of acute cerebral ischemia and have led to the design of prospective randomized clinical trials.

\section{THERAPEUTIC APPLICATIONS OF ULTRASOUND IN ACUTE CEREBRAL ISCHEMIA}

\section{Transcranial Doppler ultrasonography}

The CLOTBUST trial (which stands for Combined Lysis Of Thrombus in Brain ischemia using transcranial Ultrasound and Systemic TPA) (FIG. 1) was a phase II clinical randomized multicenter international trial with centers in Houston (United States), Barcelona (Spain), and Edmonton and Calgary (Canada). ${ }^{31}$ It had prespecified safety and signal of efficacy endpoints and a predetermined sample size of 63 patients per group. ${ }^{33}$

All enrolled patients had an acute ischemic stroke due to occlusion of the middle cerebral artery, and were treated with a standard $0.9 \mathrm{mg} / \mathrm{kg}$ dose of intravenous TPA therapy within 3 hours of symptom onset. They were randomized $(1: 1)$ to continuous TCD monitoring (Target) or placebo monitoring (Control). Emitted-power output was set at the maximal achievable level with selected insonation depths under the FDA-allowed threshold of $750 \mathrm{~mW}$. Sample volumes, or gates of insonation, were set at 3-6 $\mathrm{mm}$ for power-motion Doppler units and $10-15 \mathrm{~mm}$ for all other single-channel 2-MHz TCD units.

The safety endpoint was symptomatic intracranial hemorrhage (sICH) causing worsening of the neurologic deficit by $\geq 4$ points on the NIHSS. The primary combined activity endpoint was complete recanalization on TCD or dramatic clinical recovery by the total NIHSS score $\leq 3$ points, or improvement by $>10$ NIHSS points within 2 hours after the TPA bolus. Clinical investigators were blinded to group assignment (active or sham monitoring) done by sonographers.

All projected patients $(n=126)$ received TPA and were randomized 1:1 to target or control (with median pretreatment NIHSS score of 16 points or 17 points, respectively). Age, occlusion location on TCD, and time to TPA bolus were similar between groups. Symptomatic 


\section{Trial Transducer Tissues Exposed sICH CR mRS 0-1}

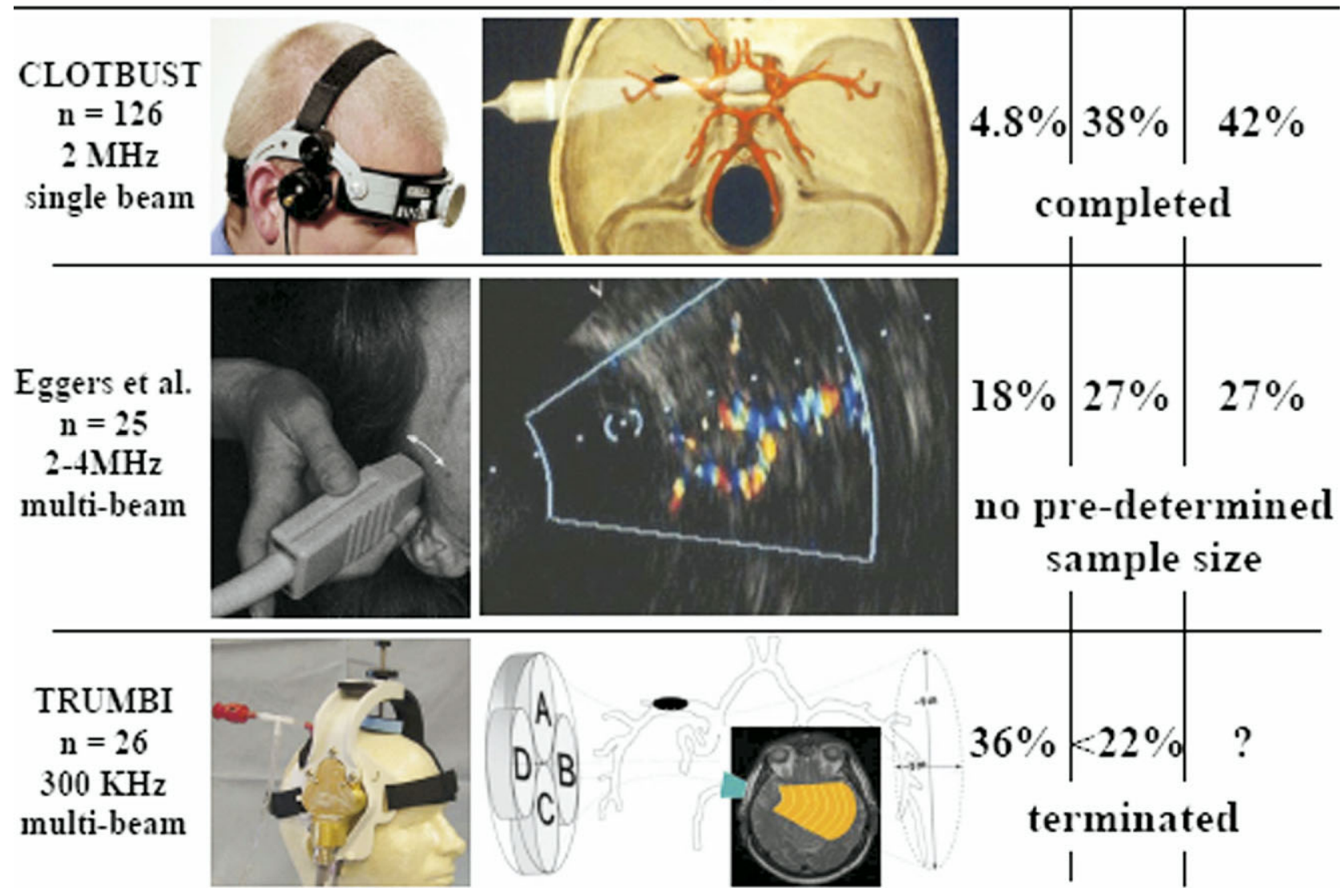

FIG. 1. Controlled clinical trials of ultrasound-enhanced systemic thrombolysis for acute ischemic stroke: Alexandrov et al. (CLOTBUST trial), ${ }^{31}$ Eggers et al., ${ }^{32}$ and Daffertshofer et al. (TRUMBI trial). ${ }^{39}$ The images under Transducer show the actual sources of ultrasound and their size relative to patient head; the images under Tissues Exposed show the beam paths as ultrasound propagates through the brain. Abbreviations: $\mathrm{CR}$, complete recanalization at the end of monitoring period; $\mathrm{kHz}$, kilohertz; $\mathrm{mRS} 0-1$, modified Rankin scores at 3 months follow-up; $n$, total number of patients enrolled in both control and target groups; $\mathrm{SICH}$, percent rates of symptomatic intracranial hemorrhages; ?, unknown (not reported in the original publication). Reproduced with permission from Alexandrov $2006 .{ }^{51}$

ICH occurred in $4.8 \%$ of Target and an equal $4.8 \%$ of Control patients. The primary endpoint was achieved by 31 Target patients (49\%) and 19 Control patients (30\%) $(P=0.03)$. At 3 months, $42 \%$ of Target and $29 \%$ of Control patients achieved favorable outcomes (mRS 0-1 points, $P=0.20$ ). This trend indicates the feasibility of a pivotal phase III clinical trial that, at 274 patients per group, would be properly powered to detect this difference in outcomes at 3 months. ${ }^{31}$

The main limitation of the CLOTBUST trial was the extreme dependence on the skill of the TCD operator, which makes these results difficult to generalize. Vascular tests that require the use of such equipment are among the most difficult to perform, and it is unrealistic to expect that an average clinician can quickly develop skills for the rapid location of occlusions with this nonimaging, hand-held diagnostic method. Hence, only a small number of stroke centers had experienced sonographers on hand before TPA therapy was begun, and thus very few qualified for the CLOTBUST and related trials.
Transcranial color-coded duplex ultrasonography

Transcranial duplex technology was tested in a smaller randomized clinical trial. ${ }^{32}$ Duplex transducers are different from those used in CLOTBUST, in that they generate multiple small beams at dual emitting frequencies, one for Doppler and one for grayscale imaging (FIG. 1). A major limitation of this technology is that there are no reliable head frames for transducer fixation, and most studies have to be performed hand-held. In addition, the mechanical index of these scanners is higher than TCD, and no dose escalation study was performed to determine how little ultrasound is needed to enhance thrombolysis without invoking safety concerns.

Eggers et al. ${ }^{32}$ evaluated 25 patients (11 Target TPA + duplex monitoring, 14 Control TPA alone) and reported a trend in the Target group toward higher recanalization rates, more hemorrhagic transformations $(18 \% \mathrm{sICH}$ rate), and better neurologic outcomes at 3 months than for patients who received TPA alone. This study did not have a predetermined sample size, and the results may 
have been affected by the small number of patients enrolled. More studies are needed to evaluate the potential of transcranial duplex technology to enhance thrombolysis.

This same group and others ${ }^{33-35}$ reported provocative findings that patients who are not eligible for systemic TPA therapy may benefit from continuous monitoring with ultrasound alone because, hypothetically, ultrasound may help facilitate the endogenous thrombolytic process that leads to spontaneous recanalization in patients with acute stroke. It is unclear if only partial recanalization can be induced by ultrasound alone, and if this exposure would result in a significant difference at 3 months, to justify a large clinical trial.

Regardless, there are no clear data regarding the benefit of ultrasound monitoring without TPA. Thus, ultrasound-alone treatment should not be substituted for TPA treatment in patients otherwise eligible for thrombolytic therapy within 3 hours of symptom onset. Furthermore, different experimental strategies are being tested in an extended time window for acute stroke treatment and continuous exposure to ultrasound may find its application in combination with other strategies such as glycoprotein IIb-IIIa antagonists, direct thrombin inhibitors.

\section{Intra-arterial ultrasound devices}

Ultrasound transducers have been incorporated into catheters for intra-arterial delivery of a thrombolytic drug (NeuroWave catheter; EKOS Corporation, Bothell, WA). This intra-arterial device uses 1.7-2.1 $\mathrm{MHz}$ pulsed-wave ultrasound with the emitting power of 400 $\mathrm{mW}$, specifications similar to extracranially applied TCD. The EKOS catheter is now being tested in phase II-III Interventional Management of Stroke (IMS) trials. $^{36}$

\section{Therapeutic low-frequency ultrasound}

In experimental models, using lower frequencies (20 $\mathrm{kHz}$ to $1 \mathrm{MHz}$ ), TPA-mediated clot degradation was as much as $50 \%$ more efficient when ultrasound was added. ${ }^{17-19}$ Consequently, it has been postulated that the use of therapeutic (i.e., nonimaging) ultrasound, in combination with intravenous thrombolytic therapy might be a feasible, safe, and potentially effective acute stroke treatment option. ${ }^{37,38}$

This hypothesis was tested in the TRUMBI trial (which stands for TRanscranial low-frequency Ultrasound-Mediated thrombolysis in Brain Ischemia). ${ }^{39}$ At first, the investigators used a very low kilohertz system $(<40 \mathrm{kHz})$, which produced intolerable tinnitus and was withdrawn from clinical testing (M. Daffertshofer, personal communication). This was replaced by a midkilohertz system operating at $300 \mathrm{kHz}$ (FIG. 1). Lowfrequency ultrasound $(300 \mathrm{kHz}, \pm 1.5 \mathrm{kHz}$ to avoid standing waves) with an intensity of $700 \mathrm{~mW} / \mathrm{cm}^{2}$ (temporal average spatial peak intensity) was applied simultaneously with intravenous administration of TPA and for 30 minutes after TPA infusion (total insonation time of 90 minutes).

The transducer had four elements arranged in a diamond pattern. The average temporal pressure was $<1$ atmosphere $(<101 \mathrm{kPa})$, thereby avoiding cavitation. The mechanical index was $<0.2$. The low intensity levels, combined with the low frequency, resulted in a thermal index in soft tissue of $<0.5$ and a cranial thermal index of $\sim 4$. The higher cranial thermal index was addressed by means of both a cooling pad and a thermal sensor to detect excessive heating. To further reduce thermal effects, ultrasound was emitted in a pulsed fashion, with a $5 \%$ duty cycle and a pulse repetition frequency of $100 \mathrm{~Hz}$ (giving a cycle/pulse ratio of 225).

The trial was terminated after 26 patients were enrolled, with a $36 \%$ rate of symptomatic hemorrhage in the Target group and no signal of efficacy on early recanalization or clinical outcomes at 3 months. The investigators found a high rate of atypical hemorrhages in the TPA-plus-ultrasound group either in the subarachnoid or in the ventricular space or at remote parenchymal locations distant to the infarct core.

The underlying mechanism causing the high rate of hemorrhage was not clear. The high rates of subarachnoid hemorrhage particularly led to speculation of some mechanical action from the ultrasound disrupting small vessels in the subarachnoid space. Further research should determine if standing pressure waves and endothelial disruption may cause these adverse effects. If confirmed in in vivo models, this will have implications on design of future kilohertz-based systems.

In conclusion, the TRUMBI trial demonstrated adverse bioeffects of mid-kilohertz ultrasound in terms of promoting bleeding both in areas affected and unaffected by ischemia.

\section{Microsphere-potentiated, ultrasound-enhanced thrombolysis}

Experimental data have suggested that ultrasound-enhanced thrombolysis can be further amplified by adding gaseous microspheres. Gaseous microspheres (safe ultrasound contrast agents) are micron-sized lipid shells that, when exposed to ultrasound, expand and produce stable cavitation with stronger reflected echoes. ${ }^{40-42}$ This is used to generate ultrasound images with better resolution. At the same time, microspheres agitate fluid where they are released by ultrasound, and this is useful in drug delivery and mechanical grinding of a thrombus. Microspheres have their own ability to lyse thrombi without a lytic drug. ${ }^{40}$ 
TABLE 1. Controlled clinical trials of microsphere-potentiated ultrasound-enhanced systemic thrombolysis for acute ischemic stroke using transcranial doppler and transcranial color-coded ultrasonography

\begin{tabular}{|c|c|c|c|c|c|c|c|}
\hline \multirow[b]{2}{*}{ Trial } & \multirow[b]{2}{*}{ Freq. } & \multirow[b]{2}{*}{ ECA } & \multirow[b]{2}{*}{ Design } & \multirow[b]{2}{*}{ Rand. } & \multicolumn{3}{|c|}{ Active Treatment Group } \\
\hline & & & & & $\mathrm{ReC}$ & AsxICH & sICH \\
\hline \multicolumn{8}{|c|}{ Transcranial Doppler ultrasonography } \\
\hline Molina et al. ${ }^{43}$ & $2 \mathrm{MHz}$ & galactose-based & $\begin{array}{l}\text { US/MS/TPA }(n=38) \text { vs. } \\
\text { US/TPA }(n=37) \text { vs. } \\
\text { TPA }(n=36)\end{array}$ & No & $71 \%$ & $23 \%$ & $3 \%$ \\
\hline $\begin{array}{l}\text { Alexandrov } \\
\text { et al. }{ }^{44}\end{array}$ & $2 \mathrm{MHz}$ & perfultren lipid & $\begin{array}{l}\text { US/MS/TPA }(n=12) \text { vs. } \\
\text { US/TPA }(n=3)\end{array}$ & Yes & $42 \%$ & $25 \%$ & $0 \%$ \\
\hline \multicolumn{8}{|c|}{ Transcranial color-coded ultrasonography } \\
\hline Larrue et al. ${ }^{46}$ & $2 \mathrm{MHz}^{\dagger}$ & galactose-based & $\begin{array}{l}\text { US/MS/TPA }(n=9) \text { vs. } \\
\text { TPA }(n=11)\end{array}$ & Yes & $48 \%$ & $78 \%$ & $0 \%$ \\
\hline Perren et al. ${ }^{45}$ & $2 \mathrm{MHz}^{\dagger}$ & $\begin{array}{l}\text { phospholipid- } \\
\text { encapsulated } \\
\mathrm{SF}_{6}\end{array}$ & $\begin{array}{l}\text { US/MS/TPA }(n=11) \text { vs. } \\
\text { TPA }(n=15)\end{array}$ & No & $64 \%$ & NA & $9 \%$ \\
\hline
\end{tabular}

Abbreviations: AsxICH, asymptomatic intracranial hemorrhage; mRS, modified Rankin Scale; MS, microspheres; NA, not available; Rand., randomization; ReC, recanalization at the end of TCD monitoring; sICH, symptomatic intracranial hemorrhage; TPA, tissue plasminogen activator; US, continuous ultrasound monitoring; ECA, echocontrast agent.

*Active treatment group: microsphere-potentiated ultrasound-enhanced systemic thrombolysis. ${ }^{\dagger}$ Patients received monitoring with a pulsedwave, 2-MHz, phased-array Doppler and intermittent exposure to dual-frequency duplex.

Several published studies have used different commercially available microspheres (TABLE 1$).{ }^{43-46}$ Molina et $\mathrm{al}^{43}$ pioneered this approach in stroke patients and reported the largest study to date that compared the CLOTBUST Target arm to the CLOTBUST Target insonation protocol (2 MHz continuous TCD-monitoring) combined with Levovist air microspheres (Schering, Berlin, Germany). Investigators demonstrated that at 2 hours after TPA bolus the TPA + TCD + Levovist group achieved a 55\% sustained recanalization rate, compared with $38 \%$ in the TPA + TCD group of the CLOTBUST trial.

The safety and feasibility of infusion of a new and more stable octofluoropropane $\left(\mathrm{C}_{3} \mathrm{~F}_{8}\right)$ perfultren lipid microspheres in patients treated with ultrasound-enhanced thrombolysis has been reported in a small phase IIA randomized clinical trial. ${ }^{43}$ Notably, in $75 \%$ of patients the microspheres permeated to areas with no pretreatment residual flow, and in $83 \%$ of patients the residual flow velocity improved at a median of 30 minutes from start of microsphere infusion (range 30 seconds120 minutes) by a median of $17 \mathrm{~cm} / \mathrm{s}$, or $118 \%$ above pretreatment values (FIG. 2). There was no sICH both in the target (TPA $+2-\mathrm{MHz}$ continuous TCDmonitoring + microspheres) and control group (TPA $+2-\mathrm{MHz}$ continuous TCD-monitoring). Moreover, after evaluation of TCD tracings as part of a secondary analysis, microspheres were moving at velocities higher than surrounding residual red blood cell flow in patients with middle cerebral artery occlusions $(39.8 \pm 11.3$ versus $28.8 \pm 13.8 \mathrm{~cm} / \mathrm{s}, P<0.001) .{ }^{47}$

Larrue et al. ${ }^{46}$ recently randomized patients with acute
( $<3$ hours) middle cerebral artery main stem occlusion as demonstrated by $\mathrm{CT}$ or magnetic resonance (MR) angiography to either transcranial duplex ultrasound continuous monitoring combined with intravenous galactose-based microspheres and TPA (combined treatment group), or TPA alone (control group). This trial was prematurely discontinued for safety reasons, because a high rate of asymptomatic intracerebral hemorrhage was demonstrated on gradient-echo MRI in the combined treatment group $(78 \%)$.

None of the intracerebral hemorrhages was symptomatic, however, and the fact that asymptomatic hemorrhagic transformation in the setting of acute cerebral ischemia has not been associated with poor outcome both in the NINDS ${ }^{48}$ and the ECASS trial ${ }^{49}$ should be taken into account when interpreting the results of the former study. It is also unclear why a data safety monitoring board was not appointed for this study and why a dose de-escalation decision was not made (i.e., reduce time of exposure to ultrasound or reduce the dose of microspheres).

In its current design, the study does not clearly indicate whether excessive hemorrhagic transformation rate was attributed to duplex ultrasound or microspheres since control subjects received TPA alone, without ultrasound. Finally, although the authors reported using continuous $2-\mathrm{MHz}$ ultrasound monitoring, the term $2-\mathrm{MHz}$ ultrasound is misleading, in that dual-frequency duplex equipment was used and, every 10 minutes during treatment, color flow, and likely B-mode, were activated to reposition the sample volume. B-mode identification of the sylvian fissure and 


\section{TPA bolus Start $\mu \mathrm{S} \quad$ Blooming}

\section{$30 \mathrm{~min}$}

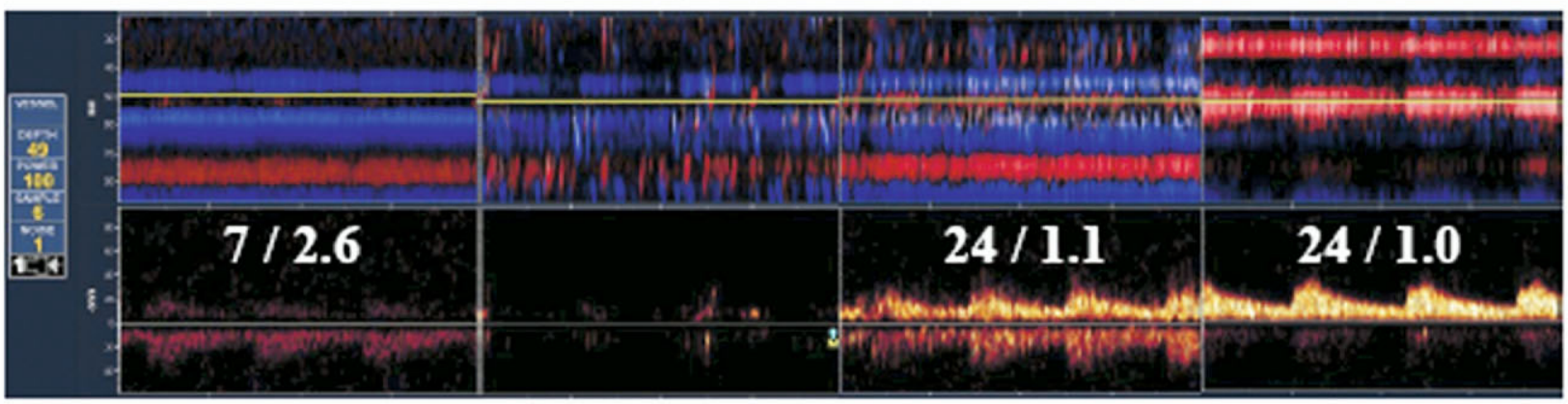

\section{$60 \mathrm{~min}$ \\ $90 \mathrm{~min}$ \\ 120 min Contralateral MCA}

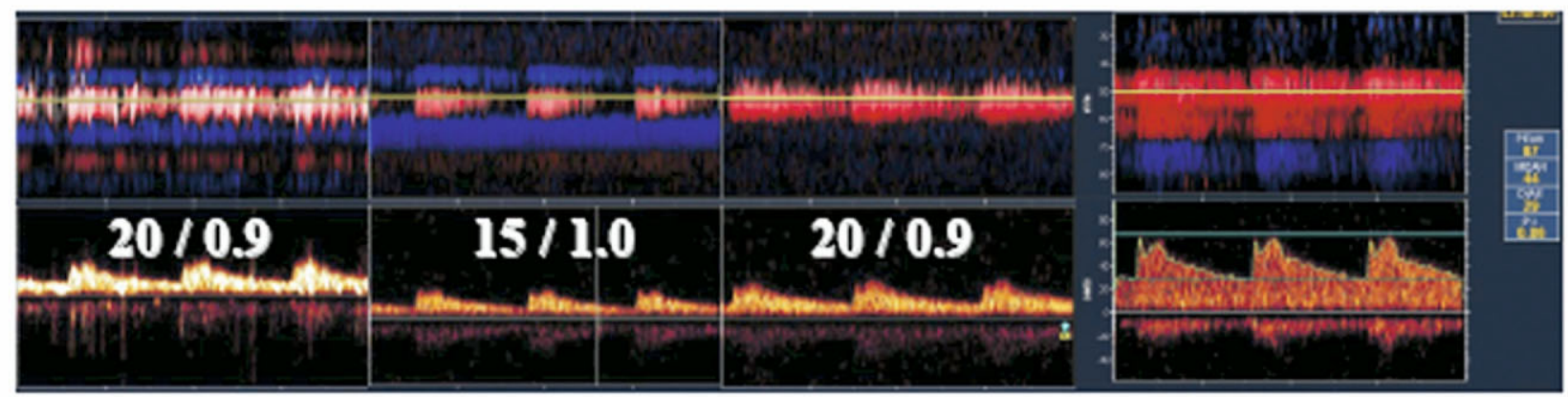

FIG. 2. Power-motion Doppler flow tracks showing individual and multiple perflutren lipid microsphere permeation to areas with no detectable residual flow pretreatment (white circles) and residual blood flow improvement following the administration of microspheres. Abbreviations: MCA, middle cerebral artery; TPA, tissue plasminogen activator; $\mu \mathrm{S}$, microsphere infusion.

other structures introduced higher frequencies during treatment. Thus, this $2-\mathrm{MHz}$ ultrasound should not be equated with other 2-MHz technologies, because by using a multitransducer phased array Doppler of essentially echocardiographic ATL (Advanced Technology Laboratories) transducer, the authors used multibeam technology that is different from TCD and that may not be completely free of other frequencies.

Finally, Perren et al ${ }^{45}$ studied the safety and feasibility of TCCD ultrasound monitoring combined with a second-generation, phospholipid-encapsulated sulfur hexafluoride $\left(\mathrm{SF}_{6}\right)$ microsphere (SonoVue; Bracco Diagnostics, Princeton, NJ) and intravenous systemic thrombolysis in patients with acute middle cerebral artery occlusion. All patients were monitored with continuous hand-held 2-MHz TCCD (pulsed-wave mode power output: $189 \mathrm{~mW} / \mathrm{cm}^{2}$; sample volume $10 \mathrm{~mm}$; Acuson Sequoia; Siemens, Malvern, PA) over $60 \mathrm{~min}-$ utes. Patients who received microsphere-potentiated ultrasound-enhanced thrombolysis seemed to fare better in terms of improvement in NIHSS score and to show sustained improvement in residual blood flow, compared with patients treated with TPA alone.

A phase I-II randomized placebo-controlled, openlabel international multicenter study (TUCSON trial) of new and more stable perfultren lipid microspheres is underway (MRX 801; Imarx Therapeutics, Tucson, AZ; http://www.imarx.com/ImaRx/clinical_trials5_0). A total of 72 patients with acute intracranial arterial occlusion as demonstrated by CT or MR angiography will be randomized to microsphere-potentiated ultrasound-enhanced thrombolysis (4 groups with increasing doses of perfultren lipid microspheres) versus TPA alone.

\section{FUTURE DIRECTIONS}

Microspheres offer a mechanical way to amplify stroke therapies, and can be developed as novel neurotherapeutics to augment brain perfusion and drug and nutrient delivery within the existing ischemic penumbra. Microspheres offer also the potential of extending the time window for therapeutic acute stroke strategies-a high-priority research prerogative. ${ }^{50}$ One obstacle to developing ultrasound and microsphere-assisted stroke therapies is the need for an experienced sonographer to locate the intracranial thrombus and expose its surface to residual flow, in order to lodge more TPA and to agitate stagnant flow. Personnel with these skills are lacking in most acute-care centers. Future studies should focus on the development of an operator-independent ultrasound device 
that can be used by existing medical personnel regardless of their experience in diagnostic ultrasound.

Acknowledgments: Dr. Tsivgoulis is the recipient of a neurosonology fellowship grant from the Neurology Department of Eginition Hospital, University of Athens School of Medicine, Athens, Greece. CLOTBUST was supported by an Investigator Sponsored Clinical Trial (from Genentech, Inc.; A2207s) and by a grant to Dr. Alexandrov from the National Institutes of Health, National Institute of Neurological Disorders and Stroke (1 K23 NS-02229-01).

\section{REFERENCES}

1. del Zoppo GJ, Poeck K, Pessin MS, et al. Recombinant tissue plasminogen activator in acute thrombotic and embolic stroke. Ann Neurol 1992;32:78-86.

2. Brott TG, Haley EC, Levy DE, et al. Urgent therapy for stroke. Part I. pilot study of tissue plasminogen activator administered within 90 minutes. Stroke 1992;23:632-640.

3. Haley EC, Levy DE, Brott TG, et al. Urgent therapy for stroke. Part II. Pilot study of tissue plasminogen activator administered 91-180 minutes from onset. Stroke 1992;23:641-645.

4. The National Institutes of Neurological Disorders and Stroke rt-PA Stroke Study Group. Tissue plasminogen activator for acute ischemic stroke. N Engl J Med 1995;333:1581-1587.

5. Hacke W, Kaste M, Fieschi C, et al. Intravenous thrombolysis with recombinant tissue plasminogen activator for acute ischemic stroke: the European Cooperative Acute Stroke Study. JAMA 1995;274:1017-1025.

6. Hacke W, Kaste M, Fieschi C, et al. Randomised double-blind placebo-controlled trial of thrombolytic therapy with intravenous alteplase in acute ischemic stroke (ECASS II): Second EuropeanAustralasian Acute Stroke Study Investigators. Lancet 1998;352: $1245-1251$.

7. Hacke W, Donnan G, Fieschi C, et al; ATLANTIS Trials Investigators; ECASS Trials Investigators; NINDS rt-PA Study Group Investigators. Association of outcome with early stroke treatment: pooled analysis of ATLANTIS, ECASS, and NINDS rt-PA stroke trials. Lancet 2004;363:768-774.

8. Haley EC, Lewandowski C, Tilley BC. Myths regarding NINDS rt-PA stroke trial: setting the record straight. Ann Emerg Med 1997;30:676-682.

9. Demchuk AM, Felberg RA, Alexandrov AV. Clinical recovery from acute ischemic stroke after early reperfusion of the brain with intravenous thrombolysis. N Engl J Med 1999;340:894-895.

10. Grotta JC, Alexandrov AV. TPA-associated reperfusion in acute ischemic stroke demonstrated by SPECT. Stroke 1998:29:429432.

11. Heiss W-D, Grond M, Thiel A, et al. Tissue at risk of infarction rescued by early reperfusion: a positron emission tomography study in systemic recombinant tissue plasminogen activator thrombolysis of acute stroke. J Cereb Blood Flow Metab 1998;18:12981307.

12. Ringelstein EB, Biniek R, Weiller C, Ammeling B, Nolte PN, Thron A. Type and extent of hemispheric brain infarctions and clinical outcome in early and delayed middle cerebral artery recanalization. Neurology 1992;42:289-298.

13. Rha JH, Saver JL. The impact of recanalization on ischemic stroke outcome: a meta-analysis. Stroke 2007;38:967-973.

14. Trubestein R, Bernard HR, Etzel F, Sobbe A, Cremer A, Stumpff U. Thrombolysis by ultrasound. Clin Sci Mol Med 1976;51:697698.

15. Tachibana K, Tachibana S. Ultrasonic vibration for boosting fibrinolytic effects of urokinase in vivo [abstract]. Thromb Haemost $1981 ; 46: 211$

16. Lauer CG, Burge R, Tang DB, Bass BG, Gomez ER, Alving BM. Effect of ultrasound on tissue-type plasminogen activator-induced thrombolysis. Circulation 1992;86:1257-1264.

17. Kimura M, Iijima S, Kobayashi K, Furuhata H. Evaluation of the thrombolytic effect of tissue-type plasminogen activator with ul- trasound irradiation: in vitro experiment involving assay of the fibrin degradation products from the clot. Biol Pharm Bull 1994;17:126-130.

18. Akiyama M, Ishibashi T, Yamada T, Furuhata H. Low-frequency ultrasound penetrates the cranium and enhances thrombolysis in vitro. Neurosurgery 1998;43:828-832.

19. Suchkova V, Siddiqi FN, Carstensen EL, Dalecki D, Child S, Francis CW. Enhancement of fibrinolysis with $40-\mathrm{kHz}$ ultrasound. Circulation 1998;98:1030-1035.

20. Behrens S, Daffertshofer M, Spiegel D, Hennerici M. Low-frequency, low-intensity ultrasound accelerates thrombolysis through the skull. Ultrasound Med Biol 1999;25:269-273.

21. Polak JF. Ultrasound energy and the dissolution of thrombus. N Engl J Med 2004;351:2154-2155.

22. Sakharov DV, Barrertt-Bergshoeff M, Hekkenberg RT, Rijken DC. Fibrin-specificity of a plasminogen activator affects the efficiency of fibrinolysis and responsiveness to ultrasound: comparison of nine plasminogen activators in vitro. Thromb Haemost 1999;81: 605-612.

23. Spengos K, Behrens S, Daffertshofer M, Dempfle CE, Hennerici $\mathrm{M}$. Acceleration of thrombolysis with ultrasound through the cranium in a flow model. Ultrasound Med Biol 2000;26:889-895.

24. Behrens S, Spengos K, Daffertshofer M, Schroeck H, Dempfle CE, Hennerici M. Transcranial ultrasound-improved thrombolysis: diagnostic vs. therapeutic ultrasound. Ultrasound Med Biol 2001;27: $1683-1689$.

25. Blinc A, Francis CW, Trudnowski JL, Carstensen EL. Characterization of ultrasound-potentiated fibrinolysis in vitro. Blood 1993; 81:2636-2643.

26. Alexandrov AV, Demchuk AM, Felberg RA, et al. High rate of complete recanalization and dramatic clinical recovery during TPA infusion when continuously monitored by $2-\mathrm{MHz}$ transcranial Doppler monitoring. Stroke 2000:31:610-614.

27. Chernyshev OY, Garami Z, Calleja S, et al. The yield and accuracy of urgent combined carotid-transcranial ultrasound testing in acute cerebral ischemia. Stroke 2005;36:32-37.

28. Tsivgoulis G, Sharma VK, Lao AY, Malkoff MD, Alexandrov AV. Validation of transcranial Doppler with computed tomography angiography in acute cerebral ischemia. Stroke 2007;38:1245-1249.

29. Kim YS, Meyer JS, Garami Z, Molina CA, Pavlovic AM, Alexandrov AV. Flow diversion in transcranial Doppler ultrasound is associated with better improvement in patients with acute middle cerebral artery occlusion. Cerebrovasc Dis 2006;21:74-78.

30. Kim YS, Garami Z, Mikulik R, Molina CA, Alexandrov AV; CLOTBUST Collaborators. Early recanalization rates and clinical outcomes in patients with tandem internal carotid artery/middle cerebral artery occlusion and isolated middle cerebral artery occlusion. Stroke 2005;36:869-871.

31. Alexandrov AV, Molina CA, Grotta JC, et al. Ultrasound-enhanced systemic thrombolysis for acute ischemic stroke. N Engl J Med 2004;351:2170-2178.

32. Eggers J, Koch B, Meyer K, Konig I, Seidel G. Effect of ultrasound on thrombolysis of middle cerebral artery occlusion. Ann Neurol 2003;53:797-800.

33. Eggers J, Seidel G, Koch B, Konig IR. Sonothrombolysis in acute ischemic stroke for patients ineligible for rt-PA. Neurology 2005; 64:1052-1054

34. Cintas P, Le Traon AP, Larrue V. High rate of recanalization of middle cerebral artery occlusion during 2-MHz transcranial colorcoded Doppler continuous monitoring without thrombolytic drug. Stroke 2002;33:626-628.

35. Skoloudik D, Bar M, Hradilek P, Vaclavik D, Skoda O. Safety and efficacy of thrombotripsy: acceleration of thrombolysis by TCCS. In: Proceedings of the NSRG 2003 Meeting, Germany: Karger [CD-ROM].

36. The IMS Study Investigators. Combined intravenous and intraarterial recanalization for acute ischemic stroke: the Interventional Management of Stroke Study. Stroke 2004;35:904-912.

37. Daffertshofer M, Hennerici M. Ultrasound in the treatment of ischaemic stroke. Lancet Neurol 2003;2:283-290.

38. Behrens S, Spengos K, Daffertshofer M, Wirth S, Hennerici M Potential use of therapeutic ultrasound in ischemic stroke treatment. Echocardiography 2001;18:259-263. 
39. Daffertshofer M, Gass A, Ringleb P, et al. Transcranial low-frequency ultrasound-mediated thrombolysis in brain ischemia: increased risk of hemorrhage with combined ultrasound and tissue plasminogen activator. Stroke 2005;36:1441-1446.

40. Unger EC, Porter T, Culp W, Labell R, Matsunaga T, Zutshi R. Therapeutic applications of lipid-coated microbubbles. Adv Drug Deliv Rev 2004;56:1291-1314.

41. Culp WC, Porter TR, McCowan TC, et al. Microbubble-augmented ultrasound declotting of thrombosed arteriovenous dialysis grafts in dogs. J Vasc Interv Radiol 2003;14:343-347.

42. Culp WC, Porter TR, Lowery J, Xie F, Roberson PK, Marky L. Intracranial clot lysis with intravenous microbubbles and transcranial ultrasound in swine. Stroke 2004;35:2407-2411.

43. Molina CA, Ribo M, Rubiera M, et al. Microbubbles administration accelerates clot lysis during continuous $2-\mathrm{MHz}$ ultrasound monitoring in stroke patients treated with intravenous tissue plasminogen activator. Stroke 2006;37:425-429.

44. Alexandrov AV, Mikulik R, Ribo M, et al. A pilot randomized clinical safety study of thrombolysis augmentation with ultrasound-activated perflutren lipid microspheres. Stroke 2007;38: LBP4 (Late Breaking Poster 4); February 2007.

45. Perren F, Loulidi J, Poglia D, Landis T, Sztajzel R. Microbubble potentiated transcranial duplex ultrasound enhances IV thrombol- ysis in acute stroke. J Thromb Thrombolysis. May 20, 2007 [Epub ahead of print].

46. Larrue V, Viguier A, Arnaud C, et al. Transcranial ultrasound combined with intravenous microbubbles and tissue plasminogen activator for acute ischemic stroke: a randomized controlled study [abstract]. Stroke 2007;38:472.

47. Sharma VK, Tsivgoulis G, Lao AY, Malkoff MD, Alexandrov AW, Alexandrov AV. Quantification of microbubble appearance in brain vessels: implications for dose calculations and drug delivery [abstract]. Stroke 2007;38:493.

48. The NINDS t-PA Stroke Study Group. Intracerebral hemorrhage after intravenous t-PA therapy for ischemic stroke. Stroke 1997; 28:2109-2118.

49. Hacke W, Kaste M, Fieschi C, et al.; for the second EuropeanAustralasian Acute Stroke Study investigators. Randomised double-blind placebo-controlled trial of thrombolytic therapy with intravenous alteplase in acute ischaemic stroke (ECASS II). Lancet 1998;352:1245-1251.

50. Meairs S, Wahlgren N, Dirnagl U, et al. Stroke research priorities for the next decade: a representative view of the European scientific community. Cerebrovasc Dis 2006;22:75-82.

51. Alexandrov AV. Ultrasound enhanced thrombolysis for stroke. Int J Stroke 2006;1:26-29. 